\title{
Research on Software Composition Algorithm for Antenna Arraying Based on Data of Chang'e
}

\author{
Juan Gao, Fei Cai, Xiaojun Cao
}

(1.School of Non-commissioned Officer of Changping, Academy of Equipment, Beijing, China;

\author{
2. 63801 of PLA, Xichang, China; \\ 3. 63713 of PLA, Taiyuan, China)
}

Key words: antenna arraying, composition algorithm, estimation of SNR

Abstract: Lunar exploration data of Chang'e-3 is collected using two measurement \& control stations 60 kilometers apart. The data is synthesized by Sumple algorithm and simulation experiments are carried out using Matlab. The synthetic efficiency is higher than $90 \%$, which verifies the applicability of the composition algorithm in remote condition.

\section{Introduction}

In space missions, especially in deep space exploration tasks, the SNR of radio signals emitted by spacecrafts may be reduced due to the effect of various complicated factors. When the SNR is lower than demodulation threshold, it is unable to receive signals properly. Therefore, how to improve the abnormal emergency handling capacity of ground based receiving equipment is a hot research at home and abroad. There are two solutions. One is increasing the area of the antenna. However, the maximum area of antenna has reached a limit. The other is synthetizing the signals received by multiple antennas, combined by arraying technology, to obtain the desired high SNR. On the premise of main technical bottleneck breakthrough, a number of countries are building their own antenna array, in order to satisfy the growing demands of deep space detection. In 2001, full spectrum group array systems has been built and put into use in the United States. The antenna arraying technology in China has also entered the stage of the ground test.

\section{Theory of Antenna Arraying}

\section{Basic Principles}

Antenna arraying is the technology using multiple antennas to receive the same signal for synthetizing. Because of deep space spacecraft signal beam in the coverage area of the ground is very wide, we can use multiple antennas, in the same area at different positions, to receive signals, and then synthesize the received signals to obtain a signal with higher $\mathrm{SNR}^{[1]}$. Naturally, the signal propagation paths and the paths between the antennas and the signal synthesizer are different. Hence the time delay between the antenna systems shall be calibrated accurately before signal synthesis. At the same time with signal synthesis, the delay jitter and phase drift shall be measured and corrected in real-time ${ }^{[2]}$. 


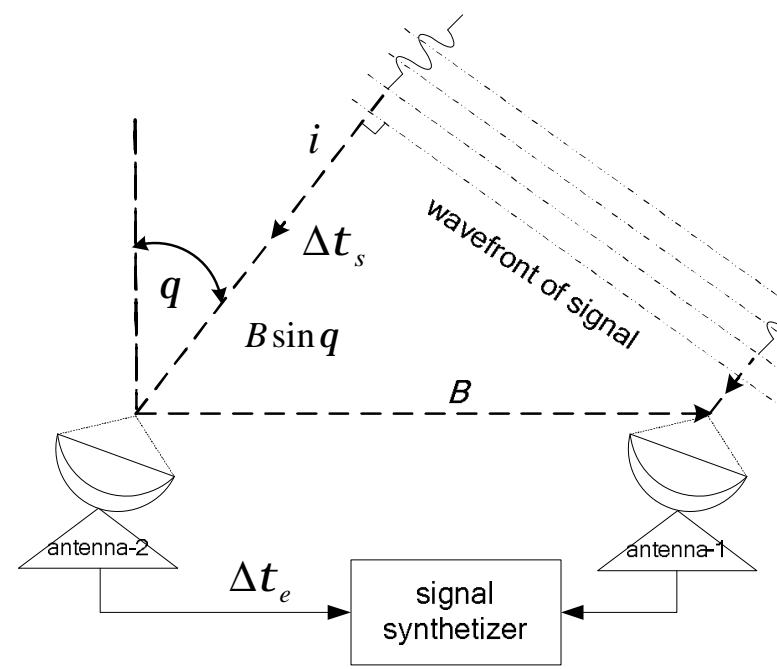

Fig.1 Schematic diagram of two antennas arraying

Arraying principle of two antennas with the same diameter is shown in fig. 1 . The time that the signal emitted by the target spacecraft required to reach the two antennas are called geometric delays. The difference between the two delays are defined as $\Delta \tau_{s}$. The difference of fixed delay of the phase center to the signal synthetizer is $\Delta \tau_{e}$. Let us suppose that the signal received by antenna-1 is:

$$
s_{1}(t)=A \sin \left(w_{s} t+\theta_{1}\right)
$$

The signal received by antenna- 2 is:

$$
s_{2}(t)=A \sin \left[w_{s}\left(t+\Delta \tau_{s}+\Delta \tau_{\varepsilon}\right)+\theta_{1}\right]=A \sin \left[w_{s} t+w_{s}\left(\Delta \tau_{s}+\Delta \tau_{\varepsilon}\right)+\theta_{1}\right]
$$

The two signals yields phase difference $w_{s}\left(\Delta \tau_{s}+\Delta \tau_{\varepsilon}\right)$. If this phase difference is compensated, the energy will be doubled by adding the two signals. On the contrary, the signal can't be enhanced, even weaker than a single signal, for instance when $w_{s}\left(\Delta \tau_{s}+\Delta \tau_{\varepsilon}\right)=\pi$ the two signals are offset.

Before each mission, $\Delta \tau_{e}$ of the antenna array shall be calibrated in high precision, then a proper delay, to offset $\Delta \tau_{e}$, is added to the transmission path between the receiver and the synthesizer.

$\Delta \tau_{s}$ can be expressed as:

$$
\Delta \tau_{s}=\frac{B \times \boldsymbol{i}}{\boldsymbol{c}}=\frac{|\boldsymbol{B}| \sin \theta}{\boldsymbol{c}}
$$

Where $\theta$ and $B$ are given, they are respectively the spacecraft observation angle and the antenna 
baseline vector. Ideally the total delay $\Delta \tau$ can be eliminated by offsetting $\Delta \tau_{e}$ and $\Delta \tau_{s}$. Hence the signal energy can be doubled by doubled by adding the two signals. In addition, if the elements of antenna array increases, the SNR of synthetized signal can be enhanced greater, it is more conductive to signal demodulation.

But in actual observation, the orbit prediction error appears, which affects the prediction accuracy of spacecraft observation angle $\theta$, and therefore results in error of $\Delta \tau_{s}$ compensation. Moreover, influenced by factors such as atmospheric turbulence, phase-shift jitter appears during transmission of signal. The factors above will degrade the performance of the arraying synthesis, therefore, we need to adopt appropriate algorithm for real-time delay jitter and phase drift correction in the process of signal synthesis.

\section{Analysis of Delay}

According to the principle mentioned above, in the post-processing, two problems need to be solved in synchronization of antenna arraying process.

1. The delay $\Delta \tau_{s}$, caused by geographic position, can be calculated by equation (3), it is compensated in arraying process, this process is called a coarse adjustment. In actual arraying process, the time-delay table, which is established based on the measured precision baseline data and track information, can be used as a look-up table for delay compensating.

2. The phase drift caused by other factors need to be compensated accurately by using arraying algorithms. This process is called a fine adjustment. The analysis and verification below are focused on fine adjustment.

\section{Performance Index}

The performance of arraying can be measured by synthetic efficiency, which is defined as ratio between real and theoretical gain of the antenna array. Suppose that the $k$ th antenna is the reference, the SNR of signal receive by each antenna is $R_{i}$, the SNR of synthetized signal is $R_{c}$. Hence the real gain is $R_{c}$ minus $R_{k}$, the theoretical gain can be obtain by adding the real gain of all antennas. The synthetize efficiency $\eta$ is given by

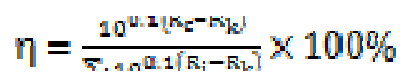

When there are only two antennas and antenna is the reference, we obtain the synthetize efficiency

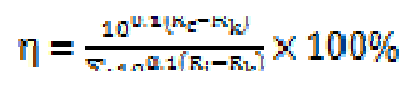

\section{Arraying Algorithms}

Arraying algorithms, such as Simple, Sumple, Eigen and Least Square, are used to determine the phase and delay offset between the elements of an antenna array. Researches demonstrated that 
Sumple is the most commonly used real-time synthesis algorithm for deep space arraying signal.

\section{Simple Algorithm}

The antenna with maximum $\mathrm{G} / \mathrm{T}$ is chosen as the reference signal, thus signals received by the else N-1 antennas are calculated with the reference. Then the phase offsets of signals are compensated using the calculation results above. Finally, weighted summation of the compensated signals is done to achieve maximum synthetic signal SNR.

Simple algorithm yields small computational complexity, do not need matrix operation, and easy for implementation. It is suitable for real-time synthesis of arraying signals. The implementation process of Simple algorithm is shown in fig. $2^{[6]}$.

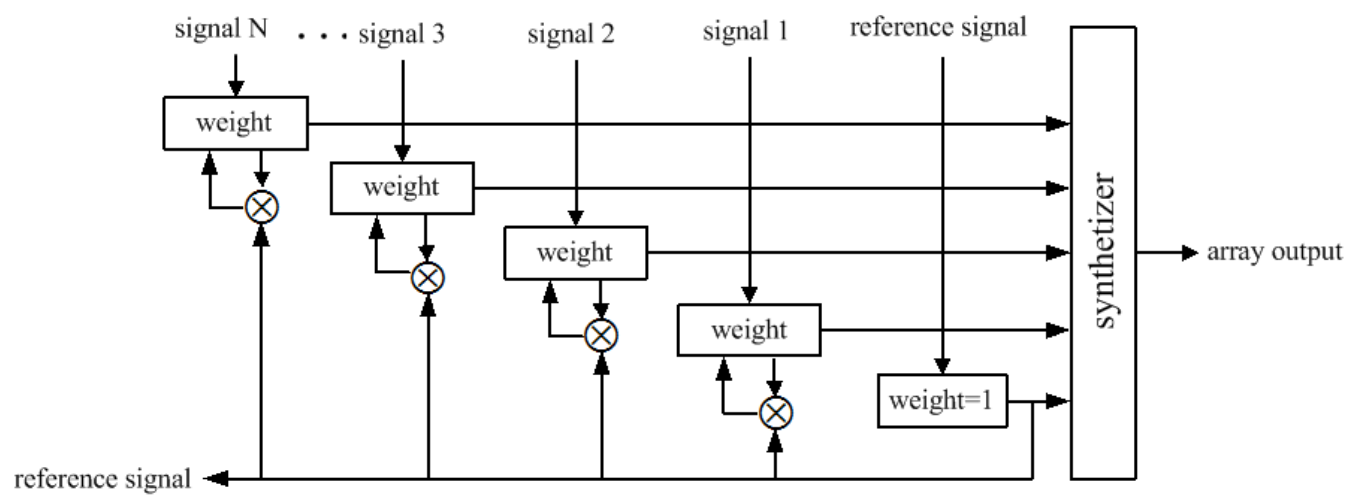

Fig.2 Schematic diagram of Simple algorithm

Simple algorithm is a kind of iterative method. It divides the signal data into many segments, then iterates the double weight obtained from the former segment to the later one, which make the signal phase difference tends to zero gradually.

Simple algorithm is suitable for real-time synthesis of deep space arraying signal. However, the disadvantages are that there is no fixed reference selection criterion for antenna arrays consist of antennas with the same diameter. If the selected reference signal is not so good or invalid, the synthetic performance will be reduced or even divergent.

\section{Sumple Algorithm}

Sumple is an improvement of Simple algorithm. Its improvement lies in that the reference antenna is the weighted summation of all antennas, namely a synthetized "antenna". The synthetized "antenna" has higher SNR, so the estimated phase shift is more accurate than that of Simple algorithm and the performance of composition is better.

Sumple algorithm is also a kind of iterative method. It divides the signal data into many segments, and then iterates the double weight obtained from the former segment to the later one. Thus matrix operation is not required, and the calculation complexity is proportional to $\mathrm{N}$. The implementation process of Sumple algorithm is shown in fig.3: 


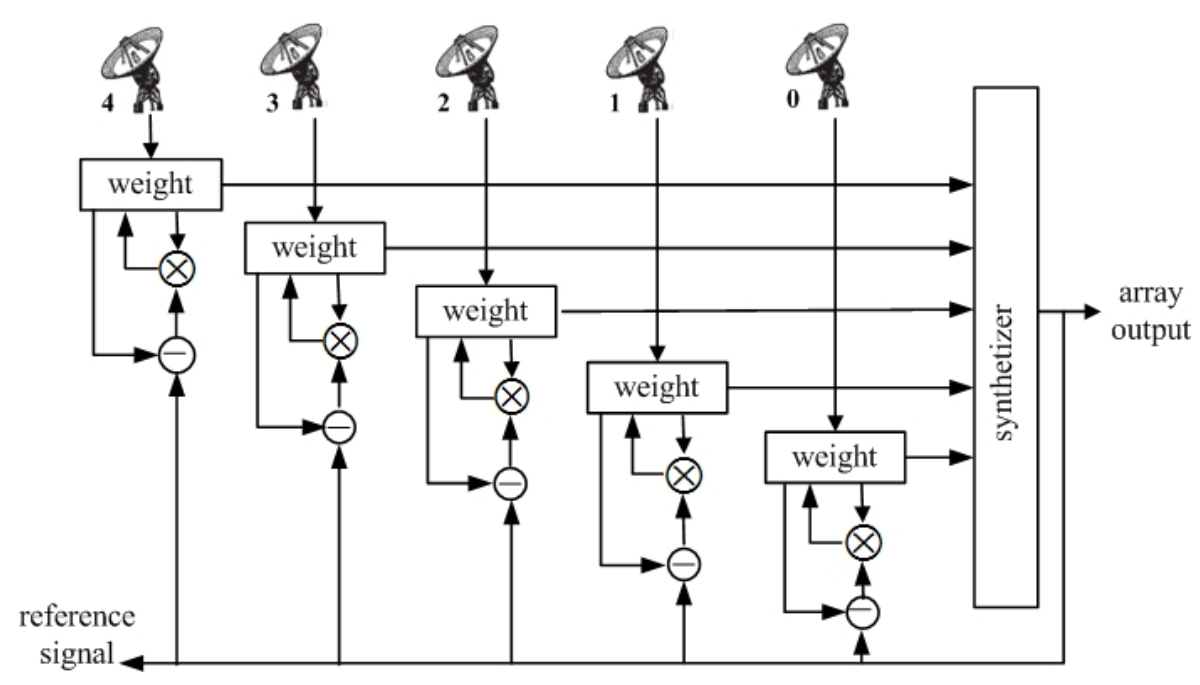

Fig.3 Schematic diagram of Sumple algorithm

\section{Experiment System}

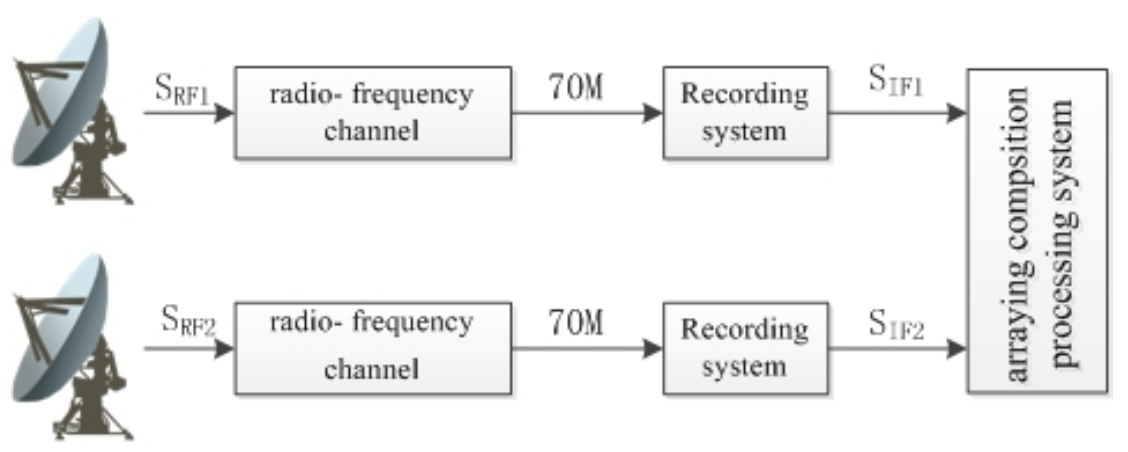

Fig.4 Experiment system

Data of Chang'e is simultaneously received by two stations 60 kilometers apart. The radiofrequency signal is converted to middle-frequency $(40 \mathrm{~Hz})$ signal by filtering and down conversion. We use a data recording system to record the data in sample rate $56 \mathrm{MHz}$, the data is stored in a hard disk for analysis and processing using the arraying composition processing system.

Sumple algorithm is used to synthetize the data of Chang'e obtained by two stations. The results will illustrate the applicability and performance of the algorithm in remote condition. The process is shown in fig.5. 


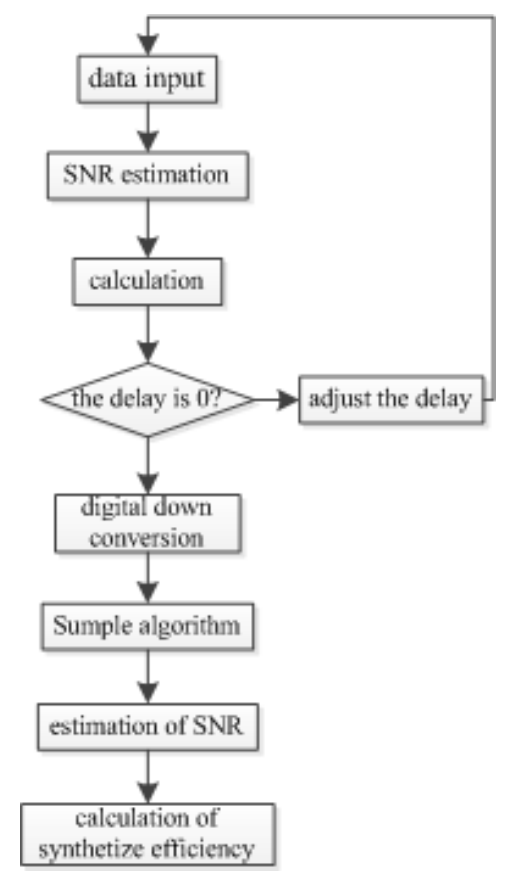

Fig.5 Process of arraying synthetizing

The data of Chang'e recorded in hard disk is transmitted to the computer, to extract the key parameters such as sampling rate, channel number and bandwidth. M2M4 algorithm is adopted to estimate the $\mathrm{SNR}^{[5]}$. Then correlation calculation of two signals is done, and the delay is adjusted according to the position of the correlation peaks. The adjusted signal is transmitted to the input of synthetize algorithm after digital down conversion (to zero). Sumple algorithm is used for phase adjustment and synthetizing, then M2M4 algorithm is adopted to estimate the SNR, hence the synthetize efficiency can be calculated using the SNR before and after composition.

According to the principle of Sumple algorithm, the sampling number of each subsection is ncor $=32768$. The channel number is Nante $=2$, the interaction number is $L=10$. The program of Sumple algorithm, written by Matlab, is as follows:

ncor=num;

Nante $=2$;

$\mathrm{Wp}=$ ones $($ Nante, $\mathrm{L}+1) ; \quad \%$ The initial phase weight is 1

$\mathrm{X}=$ zeros(Nante,num*L);

for $\mathrm{n}=1: 1:$ num $^{*} \mathrm{~L}$

$\mathrm{X}(1, \mathrm{n})=$ signalA(n);\%signalA , signalB are two signals for processing

$\mathrm{X}(2, \mathrm{n})=\operatorname{signalB}(\mathrm{n})$;

end

for $\mathrm{t}=1: \mathrm{L}$

for $\mathrm{i}=1$ :Nante

$\operatorname{Re}(\mathrm{i},:)=(1 / \mathrm{Nante}) * \mathrm{Wp}(\mathrm{i}, \mathrm{t}) * \mathrm{X}\left(\mathrm{i},(\mathrm{t}-1)^{*}\right.$ ncor+1:t*ncor $) ; \%$ weighted signals end

$\mathrm{Y}=\operatorname{sum}(\mathrm{Re})$;

$\%$ sum of weights of signals except for signals required to be reformed 


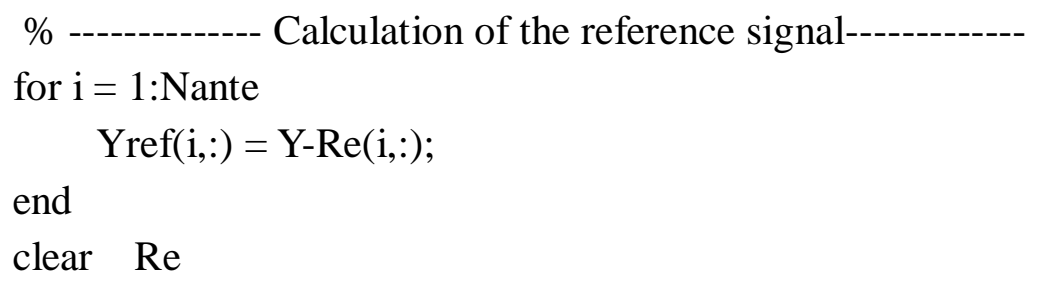
\% -------------Phase difference calculation----------------------
for $\mathrm{i}=1$ :Nante

$\mathrm{S}=\mathrm{X}\left(\mathrm{i},(\mathrm{t}-1) * \mathrm{ncor}+1: \mathrm{t}^{*}\right.$ ncor $) . * \operatorname{conj}(\operatorname{Yref}(\mathrm{i}, \mathrm{:})) ; \quad \%$ phase difference

sum_S $=\operatorname{sum}(\mathrm{S}) /$ ncor;

pAngl = atan $($ imag $($ sum_S $) . /$ real $($ sum_S $))$;

$\mathrm{Wp}(\mathrm{i}, \mathrm{t}+1)=\exp \left(-\mathrm{j}^{*} \mathrm{pAngl}\right) ; \quad \%$ estimation of phase weights

for the next stage

$\operatorname{mAngl}(\mathrm{i}, \mathrm{t})=\operatorname{pAng} 1^{*}(180 / \mathrm{pi})$

end

The spectrum of original signal is shown in fig.6.

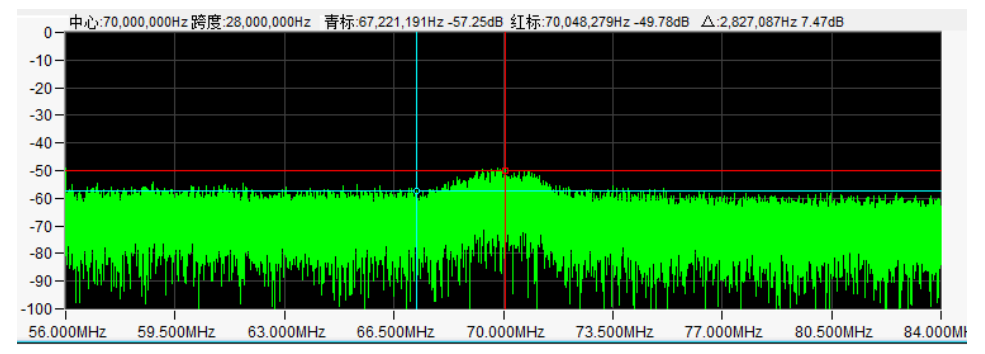

Fig.6 Spectrum of original signal

The spectrum of $56 \mathrm{MHz}$ sampled signal is illustrated in fig.7.
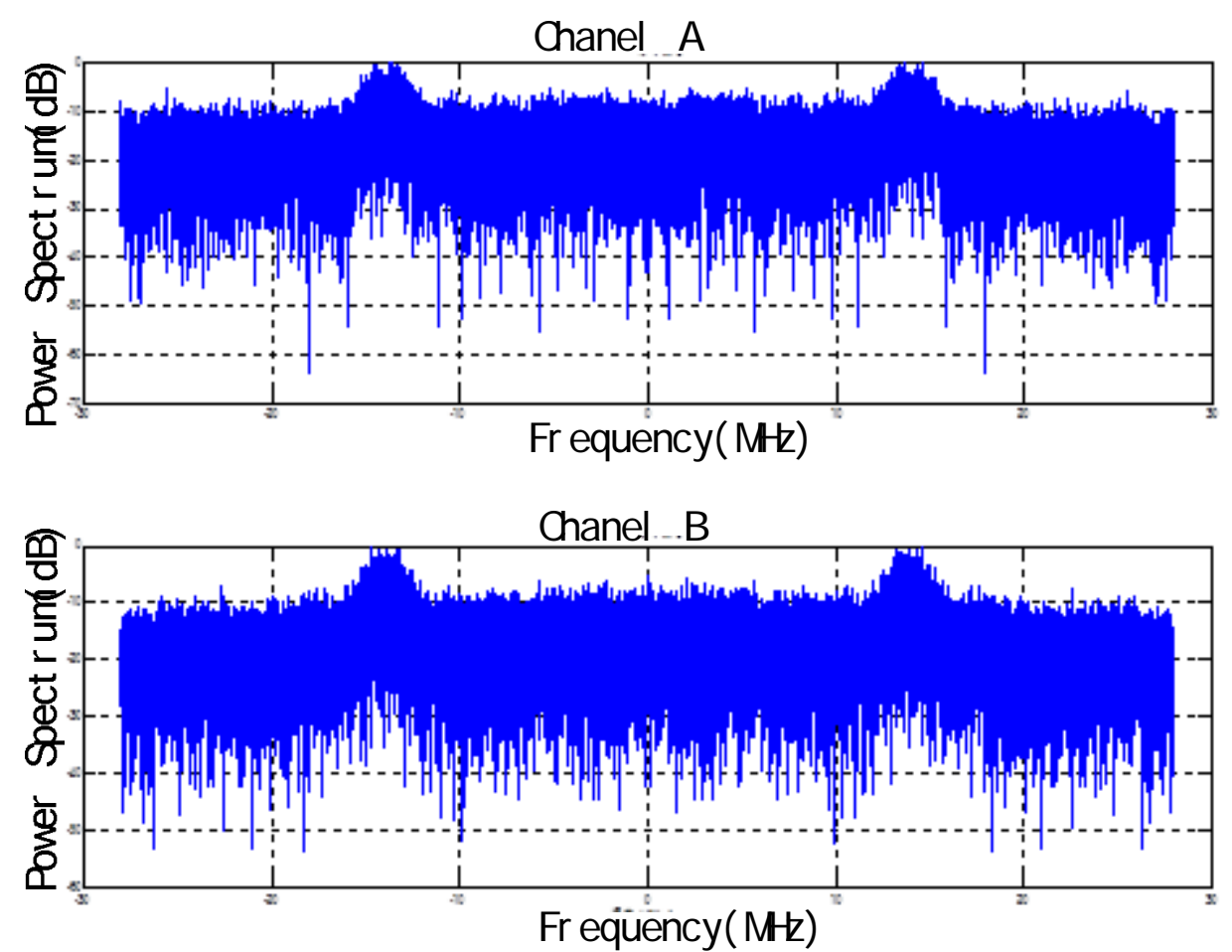

Fig.7 Spectrum of sampled signal

The spectrum of syntherized signal is shown in fig.8. The SNR of two antennas are estimated 
by $\mathrm{M} 2 \mathrm{M} 4$ algorithm, respectively $\mathrm{R}_{1}=7.02 \mathrm{~dB}$ and $\mathrm{R}_{2}=7.00 \mathrm{~dB}$. The $\mathrm{SNR}$ of reference signal is $R_{k}=8.01 \mathrm{~dB}$. The $\mathrm{SNR}$ of synthetized signal is $\mathrm{R}_{c}=9.58 \mathrm{~dB}$. We obtain the synthetize efficiency $90.4 \%$ by using equation (5).

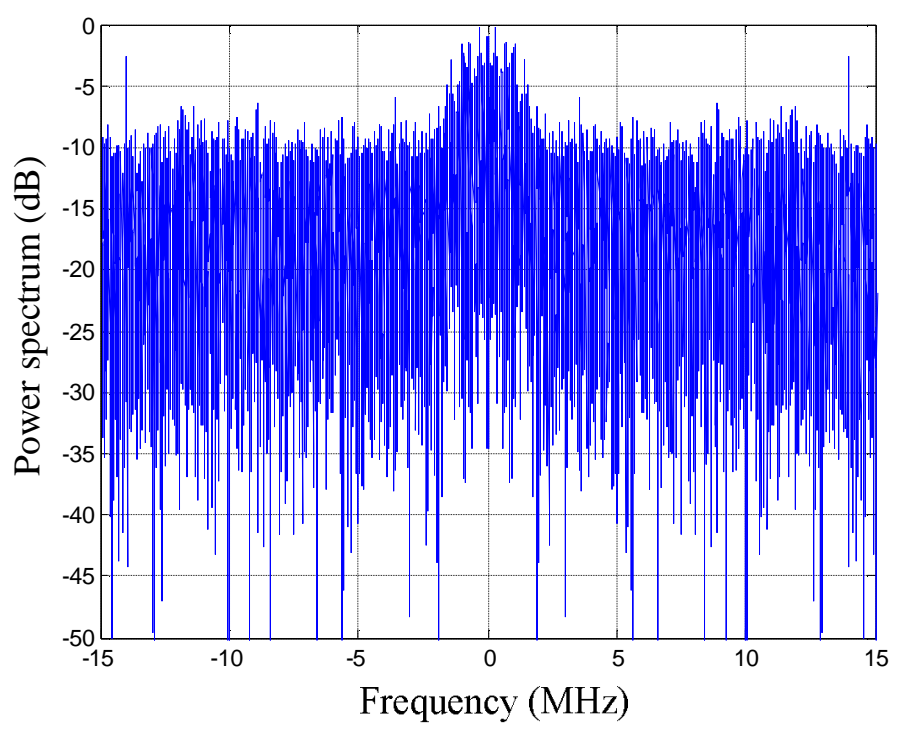

Fig. 8 Spectrum of synthetized signal

\section{Conclusions}

The algorithm proposed is verified using measured data. The results show that the synthetize efficiency of two antennas 60 kilometer apart, using Sumple algorithm, reaches $90 \%$. It illustrates that the SNR of signal receive by deep space detection can be improved greatly.

\section{References:}

[1] Hong Jiacai, Yang Wenge. Study on Downlink Antenna Array Technology and Its Test Verifying [J]. Journal of the Academy of Equipment Commend \& Technology, 2011, 22(1): 58-59.

[2] Wang Qian, Yu Hongyi. Antenna Arraying Technologies and the Development in Deep Space Network [J]. Journal of Information Engineering University, 2009, 10(3): 365-368.

[3] D. H. Rogstad. The SUMPLE Algorithm for Aligning Arrays of Receiving Radio Antennas: Coherence Achieved with Less Hardware andLowerCombining Loss [R]. IPN Progress Report. August 15, 2005

[4] Yao Fei, Kuang Linling, Zhang Yafeng, et al. Key Techniques and Development Trend of Antenna Arraying for Deep Space Communication [J]. Journal of Astronautics, 2010, 31(10): 2231-2238.

[5] Zhang Jincheng, Peng Hua, Zhao Guoqing. Research on SNR Estimation Algorithms [J]. Journal of Information Engineering University, 2011, 12(5): 535-543.

[6] D. H. Rogstad. Antenna Arraying Technologies in Deep Space Network [J]. 2005, 73-74. 
